\title{
The Ups and Downs of Coping and Sport Achievement: An Episodic Process Analysis of Within-Person Associations
}

\author{
Patrick Gaudreau, ${ }^{1}$ Adam Nicholls, ${ }^{2}$ and Andrew R. Levy ${ }^{3}$ \\ ${ }^{1}$ University of Ottawa; ${ }^{2}$ University of Hull; ${ }^{3}$ University of Leeds
}

\begin{abstract}
This study examined the relationship between coping and sport achievement at the within-person level of analysis. Fifty-four golfers completed diary measures of coping, stress, and sport achievement after six consecutive rounds of golf. Results of hierarchical linear modeling revealed golfers' episodic task-oriented coping and disengagement-oriented coping were associated, respectively, with their better and worst levels of subjective and objective achievement. Distraction-oriented coping was not significantly associated with achievement. These results were obtained after accounting for between-subjects differences in ability level and for within-person variations in perceived stress across both practice and competitive golf rounds. These results contribute to an emerging literature on the relationship between coping and sport achievement, and highlight the promises of an episodic process model of sport achievement to understand the transient self-regulatory factors associated with within-person variations in athletic achievement.
\end{abstract}

Keywords: self-regulation, sport performance, performance episodes, diary methodology, multilevel modeling

Coping is a multidimensional self-regulation construct that represents the constantly changing behavioral and cognitive mechanisms used to manage the ongoing internal and external demands of a specific stressful episode (Lazarus \& Folkman, 1984). Coping has been proposed as an essential factor to understand why some athletes perform better than others (Hardy, Jones, \& Gould, 1996). Yet, reports of significant relationships between coping variables (i.e., coping strategies, coping functions) and sport achievement remain relatively infrequent (Hoar, Kowalski, Gaudreau, \& Crocker, 2006). The aim of this research was to contribute to this scant literature by examining the relationship between coping and sport achievement from the perspective of a process-oriented approach of coping (Lazarus \& Folkman, 1984) and an episodic process model of human performance (Beal, Weiss, Barros, \& MacDermid, 2005).

Gaudreau is with the School of Psychology, University of Ottawa, Ottawa, ON, Canada. Nicholls is with the Department of Psychology, University of Hull, Hull, United Kingdom. Levy is with the Centre for Sport and Exercise Science, University of Leeds, Leeds, United Kingdom. 


\section{A Process-Oriented Model of Coping}

Several researchers have conceived coping as one of many self-regulatory processes that provide an individual with the capacity to modulate thoughts, affects, and behaviors over time and across changing environments (e.g., Compas, ConnorSmith, Saltzman, Thomsen, \& Wadsworth, 2001). Research indicates that athletes use a wide variety of coping strategies to manage the stress associated with sport competitions (Hardy et al., 1996) and hierarchical models regroup coping strategies in parsimonious higher-order dimensions of coping (e.g., Compas et al., 2001). Although labeled differently across conceptual models, two dimensions have been proposed quite systematically: task-oriented and disengagement-oriented coping (Skinner, Edge, Altman, \& Sherwood, 2003). These dimensions are based on the distinction between approach and avoidance coping (Roth \& Cohen, 1986) and, more specifically, on the difference between engagement and disengagement coping (e.g., Compas et al., 2001). Task-oriented coping represents strategies aimed at dealing directly with the stressful situation and the resulting thoughts and emotions (Skinner et al., 2003). This dimension of coping includes strategies such as effort expenditure, active coping, and thought control or cognitive reappraisal. Disengagement-oriented coping represents the strategies through which a person withdraws from the process of actively striving toward the realization of desirable outcomes, including strategies such as behavioral disengagement, denial, and venting of unpleasant emotions. In recent years, a third dimension, distraction-oriented coping, has been proposed to capture the strategies used to momentarily focus the attention on external and internal stimuli unrelated to the stressful situation (e.g., Ayers, Sandler, West, \& Roosa, 1996) and includes strategies such as distancing and mental distraction.

A recent systematic review by Nicholls and Polman (2007a) revealed that over $80 \%$ of coping studies in the sport domain were strongly influenced by the process-oriented model of coping (e.g., Lazarus \& Folkman, 1984). The notion of process centers on the unfolding flow of behaviors, affects, and cognitions within a constantly changing transaction between the person and the environment. In that regard, coping and stress are defined as transient or momentary states that may vary across time and across different situations. Research in the sport domain has lent some credence for this assumption, with the mean level of coping significantly varying across stages of a sport competition (Gaudreau, Lapierre, \& Blondin, 2001) and competitive events over a month (e.g., Nicholls \& Polman, 2007b). Moreover, recent studies with soccer players (Louvet, Gaudreau, Menaut, Genty, \& Deneuve, 2007) revealed that not all individuals change how they cope across time, thus highlighting the need to address the consequences of distinct longitudinal patterns of coping.

The process-oriented model of coping also contends that how a person copes is not a random act and that coping in a specific situation should influence the quality of outcomes in this person-environment encounter (Lazarus \& Folkman, 1984). One such outcome is achievement, which refers to the level of performance by an individual on a task as determined by objective and/or subjective criteria (VandenBos, 2007). Researchers have reported weak and inconsistent associations between coping and objective achievement such as the numbers of points in a free throw task (Haney \& Long, 1995) and the seasonal batting average of professional baseball players (Smith \& Christensen, 1995). Subjective criteria of achievement 
might be more influenced by self-regulatory processes because they involve an idiosyncratic evaluation of the amount of association/discrepancy between actual achievement and desired level of achievement (Wiese, 2007). Mounting empirical evidence suggests that coping influences subjective indicators of achievement such as goal attainment, goal progress, performance-goal discrepancy, and effectiveness (e.g., Amiot, Gaudreau, \& Blanchard, 2004; Gaudreau \& Blondin, 2004; Nicholls, Holt, Polman, \& Bloomfield, 2006). Task-oriented coping has been shown to positively relate to goal attainment and perceived coping effectiveness. In contrast, disengagement-oriented coping has been found to negatively correlate with goal attainment. Distraction-oriented coping often failed to significantly correlate with sport achievement. Nonetheless, a study with golfers indicated that distractionoriented coping could positively relate to achievement when used in combination with task-oriented forms of coping (Gaudreau \& Blondin, 2004). These results are consistent with the idea that distraction-oriented coping is a distinct dimension of coping that may allow the replenishment of limited self-regulatory resources by allowing temporary halts from the situation (Alberts, Martijn, Nievelstein, Jansen, $\&$ de Vries, 2008). These associations remained significant even after controlling for ability level, a finding of conceptual relevance given that both coping and sport achievement can differ across ability levels (Hanton, Neil, Mellalieu, \& Fletcher, 2008; Nicholls, Polman, Levy, Taylor, \& Cobley, 2007).

\section{An Episodic Model of Human Performance}

Thus far, the sport psychology literature has mainly focused on between-person associations regarding coping and achievement, rather than examining how withinperson variations in coping relate to within-person variations in achievement. The state of the literature is not surprising given that achievement has traditionally been studied in terms of between-persons differences, thus treating within-person variations as measurement error rather than the product of dynamic predictors (Dalal \& Hulin, 2008). Beal and his colleagues (2005) have recently addressed this shortcoming in a model delineating the role self-regulatory processes and affective states in within-person variations in achievement. This model centers around performance episodes, which are "naturally segmented relatively short episodes thematically organized around work-relevant immediate goals or desired end states" (p. 1055). In the sport domain, competitions can be conceived as timebounded units of performance with beginnings and endings that are subjectively experienced by sport participants, thus making them meaningful for an episodic process study of sport achievement.

The episodic model of human performance resembles the process-oriented model of Lazarus and Folkman (1984), as they both posit that within-person variations in the quality of outcomes (i.e., achievement) are mainly influenced by co-occurring self-regulatory resources and affective states. Specifically, the episodic model contends that the transient allocation of self-regulatory resources toward or away from the task at hand should respectively facilitate and hinder a person's level of achievement in a specific performance episode. This theoretical position has implications for coping research. On the one hand, task-oriented coping can be seen as the allocation of self-regulatory resources toward the specific task and resulting thoughts and emotions. On the other hand, both disengagement-oriented 
coping and distraction-oriented coping can be seen as the allocation of self-regulatory resources away from the situation. However, the role of distraction remains controversial because this coping dimension can pull a person away from the focal performance episode while allowing for the preservation and replenishment of limited self-regulatory energy (Alberts et al., 2008). ${ }^{1}$

Research in the occupational domain provided initial support for the role of coping in the episodic model of human performance. Daniels and Harris (2005) reported that daily disengagement-oriented coping of workers in public hospitals related to lower daily goal attainment whereas task-oriented coping yielded higher goal attainment on the subsequent day. Similarly, momentary deployment of efforta task-oriented coping strategy-has been linked to high levels of momentary achievement in sample of individuals measured on 50 performance episodes over a 2-wk period (Fisher \& Noble, 2004). On the basis of these promising results, it could be assumed that within-person variations in coping among sport participants may explain variations in their objective and subjective achievement across episodes of sport competitions, but research is required to explore this relationship.

\section{Hypotheses}

This diary study examined the within-person associations between coping and both subjective and objective indicators of achievement in a sample of golfers. Coping and achievement were treated as within-person variables measured after six consecutive golf rounds. Firstly, it was expected that a significant portion of the coping and sport achievement variance would be attributable to within-person fluctuations across the six events. Secondly, individuals' episodic task-oriented coping was expected to positively relate to their episodic sport achievement. Thirdly, athletes' episodic disengagement-oriented coping was hypothesized to negatively relate to their episodic sport achievement whereas distraction-oriented was expected to nonsignificantly correlate with achievement. On a final note, it was deemed crucial to control for athletes' level of ability (i.e., golf handicap) given the potential influence of this between-person variable on coping utilization (Hanton et al., 2008; Nicholls et al., 2007). Similarly, prior research revealed significant associations between perceived stress and coping (Kowalski \& Crocker, 2001; Kowalski, Crocker, Hoar, \& Niefer, 2005). Nonetheless, the relationships between coping and achievement were expected to reach significance, even while partialing out the influence of athletes' handicap and episodic perceived stress.

\section{Method}

\section{Participants}

A total of 54 male golfers from the United Kingdom completed a questionnaire after six consecutive rounds of golf. The population of golfers participating in competition is inherently heterogeneous and our convenience sampling strategy reflects this reality. The age of the golfers ranged from 18 to 83 years ( $M$ age $=50$ years, $S D=17.50$ ), and they had been playing their sport for a period ranging between 2 and 48 years $(M$ number of years $=19, S D=12)$. Their index of athletic ability (i.e., handicap) ranged from -2 to 36 , with lower values representing higher levels 
of ability. As one might expect, age and years of experience were moderately correlated $(r=.54, p<.01)$ whereas experience and handicap level were more weakly associated $(r=-.19, p>.05)$.

\section{Procedure and Measures}

Participants completed sociodemographic questions (i.e., age, golfing experience, and handicap level) before their first round of golf. The main part of the study consisted of a diary questionnaire completed after six rounds of golf: three practice rounds and three competitive rounds. There was no predetermined order in which participants completed the practice and competitive rounds questionnaires to respect the naturally occurring competitive schedule of each golfer. For instance, some athletes played one practice round followed by two competitive rounds whereas others played three practice rounds followed by three competitive rounds. Participants reported their score for the round directly on the questionnaire (i.e., stroke-play format with higher scores indicating poorer objective achievement) and they completed measures of coping, perceived stress, and subjective achievement. Each diary questionnaire was completed within $3 \mathrm{hr}$ after the round to limit the effects of memory decay on the coping data (Affleck, Zautra, Tennen, \& Armeli, 1999). Participants were asked to complete the questionnaires alone at the place of their convenience. Questionnaires were put in a sealed envelope and were returned to a research assistant at the end of the six rounds. The study was approved by the research ethic board of the university and participants provided written informed consent.

Coping. Coping was assessed using the Coping Inventory for Competitive Sport (CICS; Gaudreau \& Blondin, 2002). The CICS contains 10 subscales categorized in (1) task-oriented coping (thought control, mental imagery, relaxation, effort expenditure, logical analysis, and seeking support), (2) distraction-oriented coping (distancing and mental distraction), and (3) disengagement-oriented coping (disengagement/resignation and venting of unpleasant emotions). The CICS has nine four-item subscales and one three-item subscale, with all items rated on a scale from 1 (does not correspond at all) to 5 (corresponds very strongly). Adequate reliability has been demonstrated for the CICS with internal consistency ranging between .67 and .87 (Gaudreau \& Blondin, 2002), and support has been provided for the factor validity of the 10 -factor model as well as the three higher order dimensions (Gaudreau \& Blondin, 2002). Task- $(\alpha=.82)$, distraction- $(\alpha$ $=.68)$, and disengagement-oriented coping $(\alpha=.72)$ showed adequate level of reliability in this sample.

Perceived Stress. The stress thermometer (Kowalski \& Crocker, 2001) measured how much stress golfers had experienced in the round using a rating scale ranging from 0 (no stress) to 100 (most stress ever experienced). Prior studies have found this item to be sensitive enough to significantly correlate with coping (e.g., Kowalski et al., 2005). A single-item measure was preferred to shorten the diary to minimize missing data and attrition rates (Affleck et al., 1999), thus ensuring that stress can be used as a covariate in the analyses. 
Subjective Achievement. Three items were used to measure the extent to which golfers "attained their goal," "made progress in the pursuit of their goal," and "were satisfied about their performance while striving to attain their goal," using a scale ranging from 1 (not at all) to 9 (totally). Similar scales of subjective achievement have proven reliable in prior studies (e.g., Gaudreau \& Blondin, 2002). The measure demonstrated excellent reliability in this study $(\alpha=.94)$.

\section{Overview of Analyses}

All analyses were performed using multilevel analyses using HLM software with maximum likelihood. Multilevel analysis allows for the partitioning of the variance of a dependent variable into within-person (Level 1) and betweenperson variance (Level 2). Therefore, it was the method of choice to estimate the within-person associations, while accounting for the main effect of individual differences in athletic ability. Episodic predictors (i.e., stress and coping) were centered with the mean score of each individual to remove individual differences from the within-person associations. Level 2 predictors were centered using the grand mean centering approach to provide meaning to the intercept, which should be interpreted as the mean score of the individuals across all measurement points (Hoffmann, Griffin, \& Gavin, 2000). Multilevel analyses are suitable to handle unbalanced data when participants have completed a different number of diaries because of missing data (Affleck et al., 1999). Three participants failed to complete the measure of subjective achievement on one occasion, whereas two participants respectively did not complete it on two or three occasions. These participants could nonetheless be included in the analyses, thus resulting in a sample size of 316 at Level 1 and 54 at Level 2 for the analyses of subjective achievement. Similarly, three participants did not report their objective achievement for one round, whereas 13 participants omitted to report this information for multiple rounds. In addition, two participants did not report any of their objective achievement and they were discarded from analyses. Therefore, a sample size of 261 at Level 1 and 52 at Level 2 was used for the objective achievement analyses. ${ }^{2}$

\section{Results}

\section{Unconditional Models}

The first set of analyses tested unconditional models to estimate the portion of coping, stress, and achievement variance explained by within-person variations using the following formula: $\sigma^{2} /\left(\sigma^{2}+\tau\right)$, in which $\sigma^{2}$ represents the within-person variance and $\tau$ represents the between-person variance (see Table 1). Results indicated significant within-person variations in each of the variables $(p s<.01)$. The portion of within-person variance in coping ranged from $25 \%$ to $51 \%$, thus indicating that the golfers changed their coping utilization across the six rounds. The within-person variations explained $87 \%$ and $18 \%$ of the subjective and objective achievement variance, respectively. 


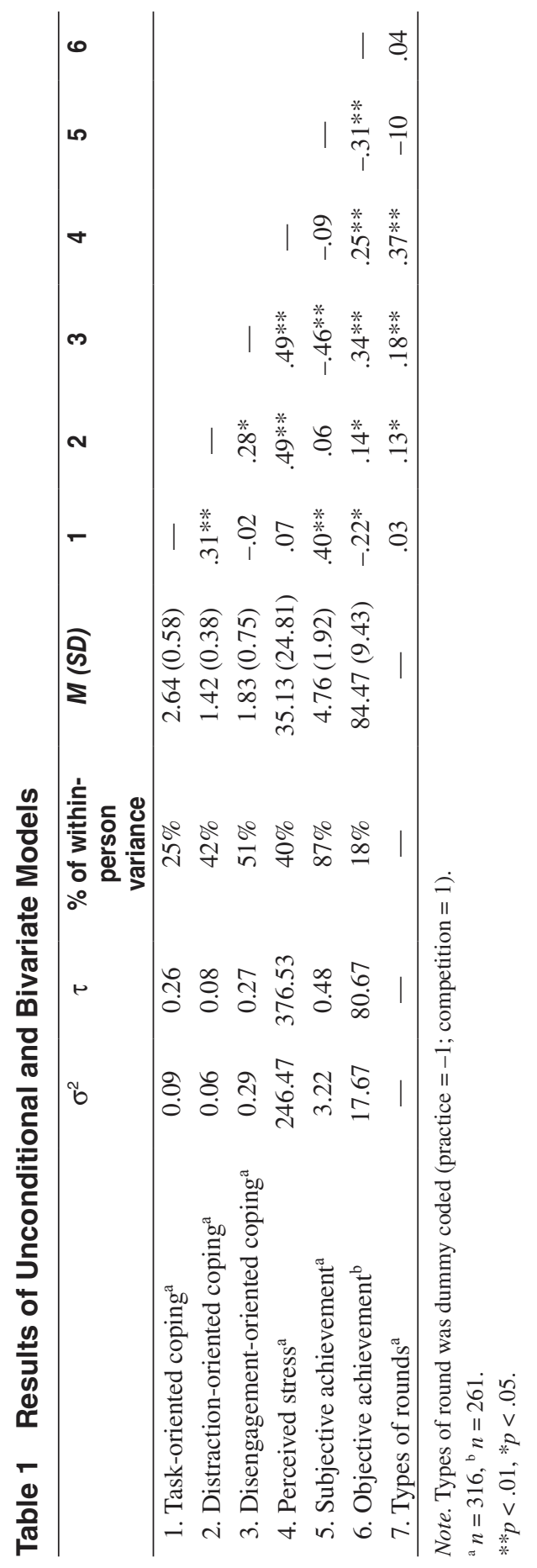




\section{Bivariate Relationships at Level 1}

A second series of analyses, examining the bivariate correlations at the within-person level of analysis (see Table 1), supported the hypothesized relationships between coping and indicators of sport achievement. Perceived stress was significantly correlated with two dimensions of coping and with objective achievement, thus confirming the need of including this variable as a covariate in the multilevel regressions.

Golfers played in three practice and three competitive rounds. Therefore, type of rounds was dummy coded (practice round $=-1$; competitive round $=1$ ) to examine its effect on the within-person scores of coping, stress, and achievement. The type of rounds was not significantly associated with achievement outcomes (see Table 1). Golfers used more disengagement- and distraction-oriented coping while experiencing higher levels of stress during competitive compared with practice rounds. Therefore, the type of rounds was included as a covariate in multilevel regression analyses.

\section{Multilevel Regression}

Two separate analyses_for subjective and objective achievement-respectively estimated the relationships of within-person variations in coping with sport achievement, while accounting for within-person variations in perceived stress and type of golf rounds (i.e., practice versus competition) using the following Level 1 equation:

$$
\begin{aligned}
\mathrm{Y} & =\pi_{0}+\pi_{1} \quad \text { (daily type of rounds) } \\
& +\pi_{2} \quad \text { (daily task) } \\
& +\pi_{3} \quad \text { (daily distraction) } \\
& \left.+\pi_{4} \quad \text { (daily disengagement }\right) \\
& +\pi_{5} \quad \text { (daily stress) } \\
& +e
\end{aligned}
$$

At the Level 2, handicap was entered to control for the main effect of individual differences in athletic ability on achievement outcomes. Random coefficients were included to let the intercept and the slope of each predictor vary across individuals, thus resulting in the Level 2 equation presented below. ${ }^{3}$

$$
\begin{gathered}
\pi_{0}=\beta_{00}+\beta_{01} \quad \text { (handicap) }+\mathrm{r}_{0} \\
\pi_{1}=\beta_{10}+\mathrm{r}_{1} \\
\pi_{2}=\beta_{20}+\mathrm{r}_{2} \\
\pi_{3}=\beta_{30}+\mathrm{r}_{3} \\
\pi_{4}=\beta_{40}+\mathrm{r}_{4} \\
\pi_{5}=\beta_{50}+\mathrm{r}_{5}
\end{gathered}
$$


Subjective Achievement. Within-person variations in task- $\left(\beta_{20}=1.80, p<\right.$ $.01)$ and disengagement-oriented coping $\left(\beta_{40}=-1.58, p<.01\right)$ were significantly associated to within-person variations in subjective achievement. In contrast, distraction-oriented coping $\left(\beta_{30}=-0.18, p>.10\right)$ was a nonsignificant predictor. These results supported the hypothesis for each of the three dimensions of coping even while considering the role of between-person differences in handicap $\left(\beta_{01}=\right.$ $-0.04, p<.01)$, within-person perceived stress $\left(\beta_{50}=-0.01, p>.10\right)$, and the type of golf round played on a specific day $\left(\beta_{10}=0.08, p>.10\right)$. The predictors accounted for $39.2 \%$ of the within-person variance in subjective achievement with $38.9 \%$ of unique variance attributable to coping.

Objective Achievement. Objective achievement was measured with the golf scores obtained in each of the six rounds, with high scores denoting poorer performance. Disengagement-oriented coping $\left(\beta_{40}=2.25, p<.01\right)$ was significantly associated with poorer objective achievement. Task-oriented coping $\left(\beta_{20}=-1.77\right.$, $p=.06$ ) was marginally significantly associated with better objective achievement during rounds of golf whereas distraction-oriented coping $\left(\beta_{30}=-1.60, p>.10\right)$ was a nonsignificant predictor. Despite the marginally significant effect of taskoriented coping, these results supported our hypotheses while controlling for the role of between-person differences in handicap $\left(\beta_{01}=0.89, p<.01\right)$, withinperson perceived stress $\left(\beta_{50}=0.05, p<.05\right)$, and the type of golf round played on a specific day $\left(\beta_{10}=-0.03, p>.10\right)$. The predictors accounted for $13.8 \%$ of the within-person variance in objective achievement with $12.4 \%$ of unique variance attributable to coping. ${ }^{4}$

Ancillary Analyses. Types of golf rounds (practice vs. competition) was included as a covariate in prior analyses. These analyses did not rule out the moderating role of practice versus competitive golf rounds. Therefore, six moderating analyses were conducted in which a specific coping dimension, the type of round, and the coping $\times$ type of round interaction were entered to predict subjective achievement and objective achievement, respectively. None of the within-person interactions reached significance ( $p s>.15)$. The within-person relationships between coping and achievement did not significantly differ across practice and competitive golf rounds. ${ }^{5}$

\section{Discussion}

Existing research on the relationships between coping and sport achievement has mainly relied on between-subject designs (e.g., Amiot et al., 2004; Gaudreau \& Blondin, 2004). This study complemented this literature by examining the withinperson relationships between coping and sport achievement within the confines of a process-oriented approach of coping (Lazarus \& Folkman, 1984) and an episodic process model of human performance (Beal et al., 2005).

Until recently, literature on the within-person variations in achievement remained scant given the lack of a general process model to guide research in this area. The model of Beal et al. (2005) recognized that an individual's achievement varies across performance episodes in a way that can be predicted by transient self-regulatory processes and affective states. Consistent with this assumption and with the work of Vancouver (1997), this study reported that subjective attainment 
largely fluctuates across performance episodes. In contrast, variations in objective golf achievement were mostly attributable to between-person differences, although the within-person variance was also significant. This finding, which is similar to diary studies outside of the sport domain (Dalal \& Hulin, 2008), is consistent with the assertion that subjective criteria of achievement are influenced to a larger extent by self-regulatory processes (Vancouver, 1997), perhaps because objective sport achievement is more strongly determined by physical, technical, tactical, and cognitive abilities.

The results of this study provide initial support for the applicability of the model of Beal et al. (2005) in the sport domain by showing that transient allocation of self-regulatory resources toward or away from the task at hand respectively facilitate and hinder a person's level of achievement in a specific performance episode. As previously discussed, objective achievement is less malleable than subjective achievement, which could explain that coping accounted for more variance in the latter indicator of sport achievement. Nonetheless, it was found that golfers' subjective and objective indicators of achievement were maximized on occasions in which they used more task-oriented coping (compared with performance episodes in which they used less task-oriented coping). In contrast, disengagement-oriented coping was associated with lower levels of both subjective and objective achievement.

Both the process-oriented approach of coping and the model of Beal et al. (2005) can be considered as multilevel models of self-regulation and human achievement. Despite a clear focus on within-person variations in coping and achievement, these models consider the influence of relatively stable between-person characteristics, such as task-relevant knowledge, skills, and personality dispositions. A stringent test of the association between episodic coping and achievement must consider the influence of stable individual differences in sport achievement. Given previous findings that highly skilled athletes might use task-oriented coping to a larger extent than their novice counterparts (e.g., Hanton et al., 2008), it was important to demonstrate a positive association between task-oriented coping and sport achievement over and above individual differences in ability level (Gaudreau \& Blondin, 2004).

In a similar vein, perceived stress is likely to influence coping (Kowalski \& Crocker, 2001; Kowalski et al., 2005). Our stringent test of the within-person association between coping and achievement accounted for the potential influence of both within-person variations in perceived stress and type of golf round played on a particular day (practice or competition) as well as between-person differences in ability level. Consequently, the associations between coping and achievement cannot be explained by a tendency of the more proficient athletes to report higher levels of achievement, nor can they be attributable to the co-occurring variations in perceived stress or to the type of round played on a given day. Nevertheless, it should be noted that the mean levels of stress, distraction-oriented coping, and disengagement-oriented coping were rather low in the current sample. This study should be interpreted as providing information about the coping and achievement relationships in situations that are not perceived as very stressful. These situations of low-to-moderate stress might also limit the need to use distraction- and disengagement-oriented coping while favoring the use of task-oriented coping. Future studies are needed with more elite athletes to replicate these findings in settings where higher levels of perceived stress might be more prevalent. 


\section{Limitations and Future Research}

Despite the desirable features of this diary design, its correlational nature precludes definitive conclusion about the direction of the coping and sport achievement relationship. An improved diary design could incorporate two measurement points for every sport event, by measuring the use of preparatory coping before each performance episode and achievement right after each encounter. Although unexplored in this study, the episodic process model also assumes that transient self-regulation can lead to "changes in the performance quality of succeeding episodes" (Beal et al., 2005, p. 1056). For instance, the results of Daniels and Harris (2005) reported that daily task-oriented coping was associated with higher goal attainment on the subsequent day. A similar study could be implemented by dividing sport competition into more precise episodes such as periods in hockey, innings in baseball, or lanes in bowling. A lead lagged design could examine whether prior coping leads to subsequent sport achievement while ruling out —or perhaps even supportingthe possibility that prior achievement leads to subsequent coping in a reciprocal or recursive manner.

This study provided evidence that the episodic relationships between coping and achievement generalize across subjective and objective indicators of achievement. Golf is a convenient sport to obtain objective sport achievement data readily comparable across all participants. Future research could collect sport achievement data via more objective routes (i.e., peer reports or official results from a competition) rather than asking participants to report their own score after each round. The self-reported procedure used in this study resulted in missing achievement data for some participants at some measurement points, thus limiting the generalizability of some of the findings. Some golfers might have been involved in match play format in which the score was not counted in a traditional way (i.e., stroke play). Other missing data might have been caused by voluntarily omitting to report bad performance or simply abandoning keeping score during the golf round. Similarly, it might be advisable to closely monitor external conditions (e.g., weather, course difficulty) likely to vary across the different rounds of golf of a diary study (Rees, Hardy, \& Freeman, 2007) and to explain some of the within-person variance in sport achievement.

Extensive and repeated self-reports could potentially interfere with the naturally occurring psychological phenomenon under investigation (Affleck et al., 1999). As such, a single-item measure of perceived stress was preferred to longer measures of perceived stress or state anxiety. This decision was deemed acceptable given that previous studies revealed significant correlations between this item and coping in the sport domain (e.g., Kowalski \& Crocker, 2001). Nonetheless, this decision prevented a more detailed examination of the associations of episodic achievement with both somatic and cognitive symptoms of anxiety. Future diary studies could incorporate short measures of state anxiety, coupled with samples of salivary cortisol, to provide a more complete assessment of various indicators associated with the stress response.

The model of Lazarus and Folkman (1984) proposes that task-oriented coping might relate to positive outcomes only when individuals perceived the situation to be controllable. Measures of cognitive appraisals (e.g., importance of the performance episode or perceived control) were not included in this study to limit the burden 
placed upon participants. Future research should examine whether the relationship between episodic coping and sport achievement is moderated by within-person variations in perceived control to examine the goodness-of-fit hypothesis (Lazarus \& Folkman,1984). Examination of such theoretically driven moderators is needed to inform sport psychologists about the social, cognitive, and motivational conditions under which task-oriented coping relates to positive outcomes. Similarly, future research should incorporate both male and female athletes to examine gender invariance of the relationships between coping and achievement, an issue of conceptual importance given the differential utilization of coping by male and female (Tamres, Janicki, \& Helgeson, 2002).

\section{Conclusion}

These results illustrate that sport psychologists and coaches should refrain from categorizing athletes with labels such as "good copers" or "bad copers." As reported in this study, a significant portion of variance in coping utilization lies within individuals rather than between individuals. Although individual differences in coping utilization exist, all athletes are at risk for falling below their usual level of achievement on days during which they rely on high disengagement-oriented coping and low task-oriented coping compared with their own average utilization. Therefore, interventions should focus on making athletes more effective in adopting, repeating, and maintaining their reliance on task-oriented coping on a momentary basis. The preference for task-oriented coping and the capacity to prevent disengagement from the goal striving process are promising skills to facilitate objective and subjective achievement.

\section{Notes}

1. The antagonistic nature of distraction could explain the nonsignificant association between distraction-oriented coping and sport achievement in the extant literature (e.g., Gaudreau \& Blondin, 2004).

2. Results of attrition analyses with ANOVAs revealed that none of the variables significantly differed $(p s>.05)$ across participants with missing points of data on objective achievement $(n=$ 18 ) and those with available data for the six rounds $(n=36)$.

3. Preliminary analyses revealed that the five slopes' random components $\left(r_{1}\right.$ to $\left.r_{5}\right)$ were nonsignificant in the model of subjective and objective achievement ( $p s>.15$ ). All tested relationships were homogeneous across participants whereas the average level of both subjective and objective achievement $\left(\mathrm{r}_{0}\right)$ had significant between-subject variability $(p<.01)$. Analyses were rerun without the random components of the slopes to yield a more parsimonious model

4. Complementary analyses were performed to examine whether within-person coping could moderate the relationships between within-person stress and both subjective and objective achievement outcomes. These analyses performed the same equations while adding three within-person interaction terms (i.e., task $\times$ stress; distraction $\times$ stress, and disengagement $\times$ stress). None of the interactions reached significance ( $p s>.10)$.

5. The coping-achievement relationships could be nonsignificant in both competition and practice rounds, while being significant after pooling the six rounds together. Such spurious relationships would be possible, even in the absence of a moderating effect, but only if both coping and achievement were significantly higher in competition compared with practice rounds (or vice 
versa). As shown in Table 1, both objective and subjective achievement were not significantly different across competitive and practice rounds, thus ruling out the potential of spurious effects.

\section{Acknowledgments}

We thank Stuart Rank, Chris Miller, and John Hughes for their help in collecting the data. This study was partially supported by a regular research grant from the Social Sciences and Humanities Research Council of Canada awarded to the first author.

\section{References}

Affleck, G., Zautra, A., Tennen, H., \& Armeli, S. (1999). Multilevel daily process designs for consulting and clinical psychology: A preface for the perplexed. Journal of Consulting and Clinical Psychology, 67, 746-754.

Alberts, H.J.E.M., Martijn, C., Nievelstein, F., Jansen, A., \& de Vries, N.K. (2008). Distracting the self: Shifting attention prevents ego depletion. Self and Identity, 7, 322-334.

Amiot, C.E., Gaudreau, P., \& Blanchard, C.M. (2004). Self-determination, coping, and goal attainment in sport. Journal of Sport \& Exercise Psychology, 26, 396-411.

Ayers, T.S., Sandler, I.N., West, S.G., \& Roosa, M.W. (1996). A dispositional and situational assessment of children's coping: Testing alternative models of coping. Journal of Personality, 64, 923-958.

Beal, D.J., Weiss, H.M., Barros, E., \& MacDermid, S.M. (2005). An episodic process model of affective influences on performance. The Journal of Applied Psychology, 90, 1054-1068.

Compas, B.E., Connor-Smith, J.K., Saltzman, H., Thomsen, A.H., \& Wadsworth, M.E. (2001). Coping with stress during childhood and adolescence: Problems, progress, and potential in theory and research. Psychological Bulletin, 127, 87-127.

Dalal, R.S., \& Hulin, C.L. (2008). Motivation for what? A multivariate dynamic perspective on the criterion. In R. Kanfer, G. Chen, \& R.D. Pritchard (Eds.), Work motivation: Past, present, and future (pp. 63-100). New York: Routledge.

Daniels, K., \& Harris, C. (2005). A daily diary study of coping in the context of the job demands-control-support model. Journal of Vocational Behavior, 66, 219-237.

Fisher, C.D., \& Noble, C.S. (2004). A within-person examination of correlates of performance and emotions while working. Human Performance, 17, 145-168.

Gaudreau, P., \& Blondin, J-P. (2002). Development of a questionnaire for the assessment of coping strategies employed by athletes in competitive sport settings. Psychology of Sport and Exercise, 3, 1-34.

Gaudreau, P., \& Blondin, J-P. (2004). The differential associations of dispositional optimism and pessimism with coping, goal attainment, and emotional adjustment during a sport competition. International Journal of Stress Management, 11, 245-269.

Gaudreau, P., Lapierre, A-M., \& Blondin, J-P. (2001). Coping at three phases of a competition: Comparison between pre-competitive, competitive, and post-competitive utilization of the same strategy. International Journal of Sport Psychology, 32, 369-385.

Haney, C.J., \& Long, B.C. (1995). Coping effectiveness: A path analysis of self-efficacy, control, coping, and performance in sport competitions. Journal of Applied Social Psychology, 25, 1726-1746.

Hanton, S., Neil, R., Mellalieu, S.D., \& Fletcher, D. (2008). Competitive experience and performance status: An investigation into multidimensional anxiety and coping. European Journal of Sport Science, 8, 143-152.

Hardy, L., Jones, G., \& Gould, D. (1996). Understanding psychological preparation for sport: Theory and practice of elite performers. Chichester, UK: Wiley. 
Hoar, S.D., Kowalski, K.C., Gaudreau, P., \& Crocker, P.R.E. (2006). A review of coping in sport. In S. Hanton \& S.D. Mellalieu (Eds.), Literature reviews in sport psychology (pp. 47-90). New York: Nova Science Publishers.

Hoffmann, D.A., Griffin, M.A., \& Gavin, M.B. (2000). The application of hierarchical linear modeling to organizational research. In K.J. Klein \& S.W.J. Kozlowski (Eds.), Multilevel theory, research, and methods in organizations: Foundations, extensions, and new directions (pp. 467-511). San Francisco, CA: Jossey-Bass.

Kowalski, K.C., \& Crocker, P.R. (2001). Development and validation of the Coping Function Questionnaire for adolescents in sport. Journal of Sport \& Exercise Psychology, 23, 136-155.

Kowalski, K.C., Crocker, P.R., Hoar, S.D., \& Niefer, C.B. (2005). Adolescents' control beliefs and coping with stress in sport. International Journal of Sport Psychology, 36, 257-272.

Lazarus, R.S., \& Folkman, S. (1984). Stress, appraisal, and coping. New York: Springer.

Louvet, B., Gaudreau, P., Menaut, A., Genty, J., \& Deneuve, P. (2007). Longitudinal patterns of stability and change in coping across three competitions: A latent class growth analysis. Journal of Sport \& Exercise Psychology, 29, 100-117.

Nicholls, A.R., Holt, N.L., Polman, R.C.J., \& Bloomfield, J. (2006). Stressors, coping, and coping effectiveness among professional rugby union players. The Sport Psychologist, 20, 314-329.

Nicholls, A.R., Polman, R., Levy, A.R., Taylor, J., \& Cobley, S. (2007). Stressors, coping, and coping effectiveness: Gender, type of sport, and skill differences. Journal of Sports Sciences, 25, 1521-1530.

Nicholls, A.R., \& Polman, R.C.J. (2007a). Coping in sport: A systematic review. Journal of Sports Sciences, 25, 11-31.

Nicholls, A.R., \& Polman, R.C.J. (2007b). Stressors, coping, and coping effectiveness among players from the England under-18 rugby union team. Journal of Sport Behavior, 30, 199-218.

Rees, T., Hardy, L., \& Freeman, P. (2007). Stressors, social support, and effects upon performance in golf. Journal of Sports Sciences, 25, 33-42.

Roth, D.L., \& Cohen, L. (1986). Approach, avoidance, and coping with stress. The American Psychologist, 41, 813-819.

Skinner, E.A., Edge, K., Altman, J., \& Sherwood, H. (2003). Searching for the structure of coping: A review and critique of category systems for classifying ways of coping. Psychological Bulletin, 129, 216-269.

Smith, R.E., \& Christensen, D.S. (1995). Psychological skills as predictors of performance and survival in professional baseball. Journal of Sport \& Exercise Psychology, 17, 399-415.

Tamres, L.K., Janicki, D., \& Helgeson, V.S. (2002). Sex differences in coping behavior: A meta-analytic review and an examination of relative coping. Personality and Social Psychology Review, 6, 2-30.

Vancouver, J.B. (1997). The application of HLM to the analysis of the dynamic interaction of environment, person, and behavior. Journal of Management, 23, 795-818.

VandenBos, G.R. (2007). APA dictionary of psychology. Washington, DC: American Psychological Association.

Wiese, B.S. (2007). Successful pursuit of personal goals and subjective well-being. In B.R. Little, K. Salmela-Aro, \& S.D. Phillips (Eds.), Personal project pursuit: Goals, action, and human flourishing (pp. 301-328). Mahwah, NJ: Lawrence Erlbaum.

Manuscript received: August 5, 2008

Revision accepted: December 5, 2009 